\title{
On the Intuitionistic Fuzzy Stability of Ring Homomorphism and Ring Derivation
}

\author{
Jaiok Roh ${ }^{1}$ and Ick-Soon Chang ${ }^{2}$ \\ ${ }^{1}$ Department of Mathematics, Hallym University, Chuncheon 200-702, Republic of Korea \\ ${ }^{2}$ Department of Mathematics, Chungnam National University, 79 Daehangno, Yuseong-gu, Daejeon 305-764, Republic of Korea
}

Correspondence should be addressed to Ick-Soon Chang; ischang@cnu.ac.kr

Received 7 June 2013; Accepted 25 July 2013

Academic Editor: Bing Xu

Copyright (c) 2013 J. Roh and I.-S. Chang. This is an open access article distributed under the Creative Commons Attribution License, which permits unrestricted use, distribution, and reproduction in any medium, provided the original work is properly cited.

We take into account the stability of ring homomorphism and ring derivation in intuitionistic fuzzy Banach algebra associated with the Jensen functional equation. In addition, we deal with the superstability of functional equation $f(x y)=x f(y)+f(x) y$ in an intuitionistic fuzzy normed algebra with unit.

\section{Introduction and Preliminaries}

The study of stability problems has originally been formulated by Ulam [1]: under what condition does there exists a homomorphism near an approximate homomorphism? Hyers [2] had answered affirmatively the question of Ulam for Banach spaces. The theorem of Hyers was generalized by Aoki [3] for additive mappings and by Rassias [4] for linear mappings by considering an unbounded Cauchy difference. The paper work of Rassias [4] has had a lot of influence in the development of what is called the generalized Hyers-Ulam stability of functional equations. Thereafter, many interesting results of the generalized Hyers-Ulam stability to a number of functional equations have been investigated. In particular, Badora [5] gave a generalization of Bourgin's result [6], and he also dealt with the stability and the Bourgin-type superstability of derivations in [7]. Recently, fuzzy version is discussed in $[8,9]$. Quite recently, the stability results in the setting of intuitionistic fuzzy normed space were studied in [10-13]; respectively, while the idea of intuitionistic fuzzy normed space was introduced in [14].

We now demonstrate some notations and basic definitions used in this work.

Definition 1 . A binary operation $*:[0,1] \times[0,1] \rightarrow[0,1]$ is said to be a continuous $t$-norm if it satisfies the following conditions:
(1) * is associative and commutative;

(2) * is continuous;

(3) $a * 1=a$ for all $a \in[0,1]$;

(4) $a * b \leq c * d$ whenever $a \leq c$ and $b \leq d$ for each $a, b, c, d \in[0,1]$.

Definition 2. A binary operation $\diamond:[0,1] \times[0,1] \rightarrow[0,1]$ is said to be a continuous $t$-conorm if it satisfies the following conditions:

(1) $\diamond$ is associative and commutative;

(2) $\diamond$ is continuous;

(3) $a \diamond 0=a$ for all $a \in[0,1]$;

(4) $a \diamond b \leq c \diamond d$ whenever $a \leq c$ and $b \leq d$ for each $a, b, c, d \in[0,1]$.

Using the notions of continuous $t$-norm and $t$-conorm, Saadati and Park [14] have recently introduced the concept of intuitionistic fuzzy normed space as follows.

Definition 3. The five-tuple $(\mathscr{X}, \mu, \nu, *, \diamond)$ is said to be an intuitionistic fuzzy normed space if $\mathscr{X}$ is a vector space, $*$ is a continuous $t$-norm, $\diamond$ is a continuous $t$-conorm, and $\mu, \nu$ are fuzzy sets on $\mathscr{X} \times(0, \infty)$ satisfying the following conditions. For every $x, y \in \mathscr{X}$ and $s, t>0$, 
(1) $\mu(x, t)+v(x, t) \leq 1$;

(2) $\mu(x, t)>0$;

(3) $\mu(x, t)=1$ if and only if $x=0$;

(4) $\mu(\alpha x, t)=\mu(\alpha x, t /|\alpha|)$ for each $\alpha \neq 0$;

(5) $\mu(x, t) * \mu(y, s) \leq \mu(x+y, t+s)$;

(6) $\mu(x, \cdot):(0, \infty) \rightarrow[0,1]$ is continuous;

(7) $\lim _{t \rightarrow \infty} \mu(x, t)=1$ and $\lim _{t \rightarrow 0} \mu(x, t)=0$;

(8) $v(x, t)<1$;

(9) $v(x, t)=0$ if and only if $x=0$;

(10) $\nu(\alpha x, t)=\nu(\alpha x, t /|\alpha|)$ for each $\alpha \neq 0$;

(11) $\nu(x, t) \diamond \mu(y, s) \geq v(x+y, t+s)$;

(12) $v(x, \cdot):(0, \infty) \rightarrow[0,1]$ is continuous;

(13) $\lim _{t \rightarrow \infty} v(x, t)=0$ and $\lim _{t \rightarrow 0} \nu(x, t)=1$.

In this case, $(\mu, \nu)$ is called an intuitionistic fuzzy norm.

Example 4. Let $(\mathscr{X},\|\cdot\|)$ be a normed space, let $a * b=a b$, and let $a \diamond b=\min \{a+b, 1\}$ for all $a, b \in[0,1]$. For all $x \in \mathscr{X}$ and every $t>0$, consider

$$
\begin{aligned}
& \mu(x, t)= \begin{cases}\frac{t}{t+\|x\|}, & \text { if } t>0, \\
0, & \text { if } t \leq 0,\end{cases} \\
& \nu(x, t)= \begin{cases}\frac{\|x\|}{t+\|x\|}, & \text { if } t>0, \\
0, & \text { if } t \leq 0 .\end{cases}
\end{aligned}
$$

Then $(\mathscr{X}, \mu, \nu, *, \diamond)$ is an intuitionistic fuzzy normed space.

Definition 5 (see [15]). The five-tuple $(\mathscr{X}, \mu, \nu, *, \diamond)$ is said to be an intuitionistic fuzzy normed algebra if $\mathscr{X}$ is an algebra, $*$ is a continuous $t$-norm, $\diamond$ is a continuous $t$-conorm, and $\mu, \nu$ are fuzzy sets on $\mathscr{X} \times(0, \infty)$ satisfying the conditions (1)-(13) of Definition 3. Furthermore, for every $x, y \in \mathscr{X}$ and $s, t>0,(14) \max \{\mu(x, t), \mu(y, s)\} \leq \mu(x y, t+s)$, (15) $\min \{v(x, t), v(y, s)\} \geq v(x y, t+s)$.

For an intuitionistic fuzzy normed algebra $(\mathscr{X}, \mu, \nu, *, \diamond)$, we further assume that (16) $a * a=a$ and $a \diamond a=a$ for all $a \in[0,1]$.

The concepts of convergence and Cauchy sequences in an intuitionistic fuzzy normed space are studied in [14]. Let $(\mathscr{X}, \mu, \nu, *, \diamond)$ be an intuitionistic fuzzy normed space or intuitionistic fuzzy normed algebra. A sequence $x=\left\{x_{k}\right\}$ is said to be intuitionistic fuzzy convergent to $L \in \mathscr{X}$ if $\lim _{k \rightarrow \infty} \mu\left(x_{k}-L, t\right)=1$ and $\lim _{k \rightarrow \infty} \nu\left(x_{k}-L, t\right)=0$ for all $t>0$. In this case, we write $(\mu, \nu)-\lim _{n \rightarrow \infty} x_{k}=L$ or $x_{k} \stackrel{I F}{\longrightarrow} L$ as $k \rightarrow \infty$. A sequence $x=\left\{x_{k}\right\}$ in $(\mathscr{X}, \mu, \nu, *, \diamond)$ is said to be intuitionistic fuzzy Cauchy sequence if $\lim _{k \rightarrow \infty} \mu\left(x_{k+p}-\right.$ $\left.x_{k}, t\right)=1$ and $\lim _{k \rightarrow \infty} \nu\left(x_{k+p}-x_{k}, t\right)=0$ for all $t>0$ and $p=$ $1,2, \ldots$. An intuitionistic fuzzy normed space or intuitionistic fuzzy normed algebra $(\mathscr{X}, \mu, \nu, *, \diamond)$ is said to be complete if every intuitionistic fuzzy Cauchy sequence in $(\mathscr{X}, \mu, \nu, *, \diamond)$ is intuitionistic fuzzy convergent in $(\mathscr{X}, \mu, \nu, *, \diamond)$. A complete intuitionistic fuzzy normed space (resp., intuitionistic fuzzy normed algebra) is also called an intuitionistic fuzzy Banach space (resp., intuitionistic fuzzy Banach algebra).

In this work, we establish the stability of ring homomorphism and ring derivation in intuitionistic fuzzy Banach algebra associated to the Jensen functional equation $2 f(x+$ $y / 2)=f(x)+f(y)$. Moreover, we consider the superstability of functional equation $f(x y)=x f(y)+f(x) y$ in intuitionistic fuzzy normed algebra with unit.

\section{The Main Results}

Remark 6. The following theorem introduced in [10] is the generalized Hyers-Ulam theorem in intuitionistic fuzzy normed space for the Jensen functional equation. However, in order to arrive at the conclusion of the theorem, the assumptions

$$
a * a \geq a, \quad a \diamond a \leq a \quad \forall a \in[0,1]
$$

should be added.

Theorem 7. Let $\mathscr{A}$ be a vector space and $f$ a mapping from $\mathscr{A}$ to an intuitionistic fuzzy Banach space $(\mathscr{B}, \mu, \nu, *, \diamond)$ with $f(0)=0$. Suppose that $\varphi$ is a function from $\mathscr{A}$ to an intuitionistic fuzzy normed space $\left(C, \mu^{\prime}, \nu^{\prime}, *, \diamond\right)$ such that

$$
\begin{gathered}
\mu\left(2 f\left(\frac{x+y}{2}\right)-f(x)-f(y), t+s\right) \\
\geq \mu^{\prime}(\varphi(x), t) * \mu^{\prime}(\varphi(y), s) \\
\nu\left(2 f\left(\frac{x+y}{2}\right)-f(x)-f(y), t+s\right) \\
\leq \nu^{\prime}(\varphi(x), t) \diamond \nu^{\prime}(\varphi(y), s)
\end{gathered}
$$

for all $x, y \in \mathscr{A} \backslash\{0\}, t>0$, and $s>0$. If $\varphi(3 x)=\alpha \varphi(x)$ for some real number $\alpha$ with $0<|\alpha|<3$, then there exists a unique additive mapping $\mathscr{L}: \mathscr{A} \rightarrow \mathscr{B}$ such that $\mathscr{L}(x):=$ $(\mu, v)-\lim _{n \rightarrow \infty}\left(f\left(3^{n} x\right) / 3^{n}\right)$,

$$
\begin{aligned}
& \mu(\mathscr{L}(x)-f(x), t) \geq M\left(x, \frac{(3-\alpha) t}{6}\right), \\
& \nu(\mathscr{L}(x)-f(x), t) \leq N\left(x, \frac{(3-\alpha) t}{6}\right),
\end{aligned}
$$

where

$$
\begin{aligned}
& M(x, t) \\
& \quad:=\mu^{\prime}\left(\varphi(x), \frac{3}{4} t\right) * \mu^{\prime}\left(\varphi(-x), \frac{3}{4} t\right) * \mu^{\prime}\left(\varphi(3 x), \frac{3}{4} t\right), \\
& N(x, t) \\
& \quad:=\nu^{\prime}\left(\varphi(x), \frac{3}{4} t\right) \diamond \nu^{\prime}\left(\varphi(-x), \frac{3}{4} t\right) \diamond \nu^{\prime}\left(\varphi(3 x), \frac{3}{4} t\right) .
\end{aligned}
$$

We begin with a generalized Hyers-Ulam theorem in intuitionistic fuzzy Banach algebra for the ring homomorphism. 
Theorem 8. Let $\mathscr{A}$ be an algebra and $f$ a mapping from $\mathscr{A}$ to an intuitionistic fuzzy Banach algebra $(\mathscr{B}, \mu, \nu, *, \diamond)$ with $f(0)=0$. Suppose that $\varphi$ is a function from $\mathscr{A}$ to an intuitionistic fuzzy normed algebra $\left(C, \mu^{\prime}, \nu^{\prime}, *, \diamond\right)$ satisfying (3) and that $\Phi$ is a function from $\mathscr{A}$ to an intuitionistic fuzzy normed space $\left(D, \mu^{\prime \prime}, \nu^{\prime \prime}, *, \diamond\right)$ such that

$$
\begin{aligned}
& \mu(f(x y)-f(x) f(y), t+s) \\
& \quad \geq \max \left\{\mu^{\prime \prime}(\Phi(x), t), \mu^{\prime \prime}(\Phi(y), s)\right\} \\
& \nu(f(x y)-f(x) f(y), t+s) \\
& \quad \leq \min \left\{v^{\prime \prime}(\Phi(x), t), \nu^{\prime \prime}(\Phi(y), s)\right\}
\end{aligned}
$$

for all $x, y \in \mathscr{A}, t>0$, and $s>0$. If $\varphi(3 x)=\alpha \varphi(x)$ for some real number $\alpha$ with $0<|\alpha|<3$ and $\Phi(3 x)=\beta \Phi(x)$ for some real number $\beta$ with $0<|\beta|<3$, then there exists a unique ring homomorphism $\mathscr{L}: \mathscr{A} \rightarrow \mathscr{B}$ satisfying (4).

Proof. It follows by Theorem 7 that there exists a unique additive mapping $\mathscr{L}: \mathscr{A} \rightarrow \mathscr{B}$ satisfying $(4)$, where $\mathscr{L}(x):=$ $(\mu, v)-\lim _{n \rightarrow \infty}\left(f\left(3^{n} x\right) / 3^{n}\right)$.

Without loss of generality, we suppose that $0<\beta<3$. We prove that $\mathscr{L}$ is a ring homomorphism. Note that

$$
\begin{aligned}
\mu(\mathscr{L}(x y)-\mathscr{L}(x) f(y), t) & \\
\geq & \left(\mathscr{L}(x y)-\frac{f\left(3^{n} x y\right)}{3^{n}}, \frac{t}{3}\right) \\
& * \mu\left(\frac{f\left(3^{n} x y\right)}{3^{n}}-\frac{f\left(3^{n} x\right)}{3^{n}} f(y), \frac{t}{3}\right) \\
& * \mu\left(\frac{f\left(3^{n} x\right)}{3^{n}} f(y)-\mathscr{L}(x) f(y), \frac{t}{3}\right), \\
\nu(\mathscr{L}(x y)-\mathscr{L}(x) f(y), t) & \left(\mathscr{L}(x y)-\frac{f\left(3^{n} x y\right)}{3^{n}}, \frac{t}{3}\right) \\
\leq & \diamond \nu\left(\frac{f\left(3^{n} x y\right)}{3^{n}}-\frac{f\left(3^{n} x\right)}{3^{n}} f(y), \frac{t}{3}\right) \\
\diamond & \mu\left(\frac{f\left(3^{n} x\right)}{3^{n}} f(y)-\mathscr{L}(x) f(y), \frac{t}{3}\right)
\end{aligned}
$$

for all $x, y \in \mathscr{A}$ and $t>0$. On the other hand, we see that

$$
\begin{aligned}
& \mu\left(\frac{f\left(3^{n} x \cdot y\right)}{3^{n}}-\frac{f\left(3^{n} x\right)}{3^{n}} f(y), \frac{t}{3}\right) \\
& \quad=\mu\left(f\left(3^{n} x y\right)-f\left(3^{n} x\right) f(y), 3^{n-1} t\right) \\
& \quad \geq \max \left\{\mu^{\prime \prime}\left(\Phi(x),\left(\frac{3}{\beta}\right)^{n} \frac{t}{6}\right), \mu^{\prime \prime}\left(\Phi(y), \frac{3^{n-1} t}{2}\right)\right\},
\end{aligned}
$$

$$
\begin{aligned}
\nu\left(\frac{f\left(3^{n} x \cdot y\right)}{3^{n}}-\frac{f\left(3^{n} x\right)}{3^{n}} f(y), \frac{t}{3}\right) \\
\quad=v\left(f\left(3^{n} x y\right)-f\left(3^{n} x\right) f(y), 3^{n-1} t\right) \\
\quad \leq \min \left\{v^{\prime \prime}\left(\Phi(x),\left(\frac{3}{\beta}\right)^{n} \frac{t}{6}\right), \nu^{\prime \prime}\left(\Phi(y), \frac{3^{n-1} t}{2}\right)\right\}
\end{aligned}
$$

for all $x, y \in \mathscr{A}$ and $t>0$ and

$$
\begin{aligned}
& \mu\left(\frac{f\left(3^{n} x\right)}{3^{n}} f(y)-\mathscr{L}(x) f(y), \frac{t}{3}\right) \\
& =\mu\left(f\left(3^{n} x\right) f(y)-3^{n} \mathscr{L}(x) f(y), 3^{n-1} t\right) \\
& \geq \max \left\{\mu\left(\frac{f\left(3^{n} x\right)}{3^{n}}-\mathscr{L}(x), \frac{t}{6}\right),\right. \\
& \left.\mu\left(f(y), \frac{3^{n-1} t}{2}\right)\right\}, \\
& \nu\left(\frac{f\left(3^{n} x\right)}{3^{n}} f(y)-\mathscr{L}(x) f(y), \frac{t}{3}\right) \\
& =\nu\left(f\left(3^{n} x\right) f(y)-3^{n} \mathscr{L}(x) f(y), 3^{n-1} t\right) \\
& \leq \min \left\{\nu\left(\frac{f\left(3^{n} x\right)}{3^{n}}-\mathscr{L}(x), \frac{t}{6}\right), \nu\left(f(y), \frac{3^{n-1} t}{2}\right)\right\}
\end{aligned}
$$

for all $x, y \in \mathscr{A}$ and $t>0$. Letting $n \rightarrow \infty$ in (7), (8), and (9), we get

$$
\begin{aligned}
& \mu(\mathscr{L}(x y)-\mathscr{L}(x) f(y), t)=1, \\
& \nu(\mathscr{L}(x y)-\mathscr{L}(x) f(y), t)=0 .
\end{aligned}
$$

This implies that

$$
\mathscr{L}(x y)=\mathscr{L}(x) f(y)
$$

for all $x, y \in \mathscr{A}$.

Using additivity of $\mathscr{L}$ and (11), we find that

$$
\begin{aligned}
3^{n} \mathscr{L}(x) f(y) & =\mathscr{L}\left(3^{n} x \cdot y\right) \\
& =\mathscr{L}\left(x \cdot 3^{n} y\right)=\mathscr{L}(x) f\left(3^{n} y\right) .
\end{aligned}
$$

So we obtain $\mathscr{L}(x) f(y)=\mathscr{L}(x)\left(f\left(3^{n} y\right) / 3^{n}\right)$; that is,

$$
\begin{gathered}
\mu\left(\mathscr{L}(x) f(y)-\mathscr{L}(x) \frac{f\left(3^{n} y\right)}{3^{n}}, t\right)=1, \\
\nu\left(\mathscr{L}(x) f(y)-\mathscr{L}(x) \frac{f\left(3^{n} y\right)}{3^{n}}, t\right)=0
\end{gathered}
$$


for all $x, y \in \mathscr{A}$ and $t>0$. This relation yields that

$$
\begin{aligned}
& \mu(\mathscr{L}(x) f(y)-\mathscr{L}(x) \mathscr{L}(y), t) \\
& \geq \mu\left(\mathscr{L}(x) f(y)-\mathscr{L}(x) \frac{f\left(3^{n} y\right)}{3^{n}}, \frac{t}{2}\right) \\
& * \mu\left(\mathscr{L}(x) \frac{f\left(3^{n} y\right)}{3^{n}}-\mathscr{L}(x) \mathscr{L}(y), \frac{t}{2}\right) \\
& =\mu\left(\mathscr{L}(x) \frac{f\left(3^{n} y\right)}{3^{n}}-\mathscr{L}(x) \mathscr{L}(y), \frac{t}{2}\right), \\
& v(\mathscr{L}(x) f(y)-\mathscr{L}(x) \mathscr{L}(y), t) \\
& \leq v\left(\mathscr{L}(x) f(y)-\mathscr{L}(x) \frac{f\left(3^{n} y\right)}{3^{n}}, \frac{t}{2}\right) \\
& \diamond v\left(\mathscr{L}(x) \frac{f\left(3^{n} y\right)}{3^{n}}-\mathscr{L}(x) \mathscr{L}(y), \frac{t}{2}\right) \\
& =v\left(\mathscr{L}(x) \frac{f\left(3^{n} y\right)}{3^{n}}-\mathscr{L}(x) \mathscr{L}(y), \frac{t}{2}\right)
\end{aligned}
$$

for all $x, y \in \mathscr{A}$ and $t>0$. On the other hand, we see that

$$
\begin{aligned}
& \mu\left(\mathscr{L}(x) \frac{f\left(3^{n} y\right)}{3^{n}}-\mathscr{L}(x) \mathscr{L}(y), \frac{t}{2}\right) \\
&=\mu\left(\mathscr{L}(x) f\left(3^{n} y\right)-3^{n} \mathscr{L}(x) \mathscr{L}(y), \frac{3^{n} t}{2}\right) \\
& \geq \max \left\{\mu\left(\mathscr{L}(x), \frac{3^{n} t}{4}\right), \mu\left(\frac{f\left(3^{n} y\right)}{3^{n}}-\mathscr{L}(y), \frac{t}{4}\right)\right\}, \\
& v\left(\mathscr{L}(x) \frac{f\left(3^{n} y\right)}{\left.3^{n}-\mathscr{L}(x) \mathscr{L}(y), \frac{t}{2}\right)}\right. \\
& \quad=v\left(\mathscr{L}(x) f\left(3^{n} y\right)-3^{n} \mathscr{L}(x) \mathscr{L}(y), \frac{3^{n} t}{2}\right) \\
& \leq \min \left\{v\left(\mathscr{L}(x), \frac{3^{n} t}{4}\right), v\left(\frac{f\left(3^{n} y\right)}{3^{n}}-\mathscr{L}(y), \frac{t}{4}\right)\right\}
\end{aligned}
$$

for all $x, y \in \mathscr{A}$ and $t>0$. Sending $n \rightarrow \infty$ in (14) and (15), we have

$$
\begin{gathered}
\mu(\mathscr{L}(x) f(y)-\mathscr{L}(x) \mathscr{L}(y), t)=1, \\
\nu(\mathscr{L}(x) f(y)-\mathscr{L}(x) \mathscr{L}(y), t)=0 .
\end{gathered}
$$

Thus, we conclude that

$$
\mathscr{L}(x) f(y)=\mathscr{L}(x) \mathscr{L}(y)
$$

for all $x, y \in \mathscr{A}$.

Therefore, by combining (11) and (17), we get $\mathscr{L}(x y)=$ $\mathscr{L}(x) \mathscr{L}(y)$, which completes the proof.

Now we recall that an additive mapping $d$ on an algebra $\mathscr{A}$ is said to be a ring derivation if the functional equation $d(x y)=x d(y)+d(x) y$ holds for all $x, y \in \mathscr{A}$.
Theorem 9. Let $(\mathscr{A}, \mu, \nu, *, \diamond)$ be an intuitionistic fuzzy Banach algebra and $f: \mathscr{A} \rightarrow \mathscr{A}$ a mapping with $f(0)=$ 0 . Assume that $\varphi$ is a function from $\mathscr{A}$ to an intuitionistic fuzzy normed space $\left(C, \mu^{\prime}, v^{\prime}, *, \diamond\right)$ satisfying (3) and that $\Phi$ is a function from $\mathscr{A}$ to an intuitionistic fuzzy normed space $\left(D, \mu^{\prime \prime}, v^{\prime \prime}, *, \diamond\right)$ such that

$$
\begin{aligned}
& \mu(f(x y)-x f(y)-f(x) y, t+s) \\
& \quad \geq \max \left\{\mu^{\prime \prime}(\Phi(x), t), \mu^{\prime \prime}(\Phi(y), s)\right\}, \\
& \nu(f(x y)-x f(y)-f(x) y, t+s) \\
& \quad \leq \min \left\{v^{\prime \prime}(\Phi(x), t), \nu^{\prime \prime}(\Phi(y), s)\right\}
\end{aligned}
$$

for all $x, y \in \mathscr{A}, t>0$, and $s>0$. If $\varphi(3 x)=\alpha \varphi(x)$ for some real number $\alpha$ with $0<|\alpha|<3$ and $\Phi(3 x)=\beta \Phi(x)$ for some real number $\beta$ with $0<|\beta|<3$, then there exists a unique ring derivation $\mathscr{L}: \mathscr{A} \rightarrow \mathscr{A}$ satisfying (4). Moreover,

$$
x\{f(y)-\mathscr{L}(y)\}=0
$$

is fulfilled for all $x, y \in \mathscr{A}$.

Proof. By Theorem 7, there exists a unique additive mapping $\mathscr{L}: \mathscr{A} \rightarrow \mathscr{B}$ satisfying (4), where $\mathscr{L}(x):=(\mu, v)-$ $\lim _{n \rightarrow \infty}\left(f\left(3^{n} x\right) / 3^{n}\right)$.

As in the proof of Theorem 8, we consider $0<|\beta|<3$. We show that $\mathscr{L}$ is a ring derivation. Observe that

$$
\begin{aligned}
& \mu(\mathscr{L}(x y)-x f(y)-\mathscr{L}(x) y, t) \\
& \geq \mu\left(\mathscr{L}(x y)-\frac{f\left(3^{n} x y\right)}{3^{n}}, \frac{t}{3}\right) \\
& * \mu\left(\frac{f\left(3^{n} x y\right)}{3^{n}}-x f(y)-\frac{f\left(3^{n} x\right)}{3^{n}} y, \frac{t}{3}\right) \\
& * \mu\left(\frac{f\left(3^{n} x\right)}{3^{n}} y-\mathscr{L}(x) y, \frac{t}{3}\right), \\
& v(\mathscr{L}(x y)-x f(y)-\mathscr{L}(x) y, t) \\
& \leq v\left(\mathscr{L}(x y)-\frac{f\left(3^{n} x y\right)}{3^{n}}, \frac{t}{3}\right) \\
& \diamond v\left(\frac{f\left(3^{n} x y\right)}{3^{n}}-x f(y)-\frac{f\left(3^{n} x\right)}{3^{n}} y, \frac{t}{3}\right) \\
& \diamond v\left(\frac{f\left(3^{n} x\right)}{3^{n}} y-\mathscr{L}(x) y, \frac{t}{3}\right)
\end{aligned}
$$

for all $x, y \in \mathscr{A}$ and $t>0$. On the other hand, we yield that

$$
\begin{aligned}
& \mu\left(\frac{f\left(3^{n} x y\right)}{3^{n}}-x f(y)-\frac{f\left(3^{n} x\right)}{3^{n}} y, \frac{t}{3}\right) \\
& \quad=\mu\left(f\left(3^{n} x \cdot y\right)-3^{n} x \cdot f(y)-f\left(3^{n} x\right) y, 3^{n-1} t\right) \\
& \quad \geq \max \left\{\mu^{\prime \prime}\left(\Phi(x),\left(\frac{3}{\beta}\right)^{n} \frac{t}{6}\right), \mu^{\prime \prime}\left(\Phi(y), \frac{3^{n-1} t}{2}\right)\right\},
\end{aligned}
$$




$$
\begin{aligned}
& \nu\left(\frac{f\left(3^{n} x y\right)}{3^{n}}-x f(y)-\frac{f\left(3^{n} x\right)}{3^{n}} y, \frac{t}{3}\right) \\
& \quad=v\left(f\left(3^{n} x \cdot y\right)-3^{n} x \cdot f(y)-f\left(3^{n} x\right) y, 3^{n-1} t\right) \\
& \quad \leq \min \left\{v^{\prime \prime}\left(\Phi(x),\left(\frac{3}{\beta}\right)^{n} \frac{t}{6}\right), v^{\prime \prime}\left(\Phi(y), \frac{3^{n-1} t}{2}\right)\right\}
\end{aligned}
$$

for all $x, y \in \mathscr{A}$ and $t>0$ and

$$
\begin{aligned}
& \mu\left(\frac{f\left(3^{n} x\right)}{3^{n}} y-\mathscr{L}(x) y, \frac{t}{3}\right) \\
& \quad=\mu\left(f\left(3^{n} x\right) y-3^{n} \mathscr{L}(x) y, 3^{n-1} t\right) \\
& \quad \geq \max \left\{\mu\left(\frac{f\left(3^{n} x\right)}{3^{n}}-\mathscr{L}(x), \frac{t}{6}\right), \mu\left(y, \frac{3^{n-1} t}{2}\right)\right\}, \\
& \nu\left(\frac{f\left(3^{n} x\right)}{3^{n}} y-\mathscr{L}(x) y, \frac{t}{3}\right) \\
& \quad=v\left(f\left(3^{n} x\right) y-3^{n} \mathscr{L}(x) y, 3^{n-1} t\right) \\
& \quad \leq \min \left\{v\left(\frac{f\left(3^{n} x\right)}{3^{n}}-\mathscr{L}(x), \frac{t}{6}\right), \nu\left(y, \frac{3^{n-1} t}{2}\right)\right\}
\end{aligned}
$$

for all $x, y \in \mathscr{A}$ and $t>0$. Letting $n \rightarrow \infty$ in (20), (21), and (22), we have

$$
\begin{aligned}
& \mu(\mathscr{L}(x y)-x f(y)-\mathscr{L}(x) y, t)=1, \\
& \nu(\mathscr{L}(x y)-x f(y)-\mathscr{L}(x) y, t)=0 .
\end{aligned}
$$

So we get

$$
\mathscr{L}(x y)=x f(y)+\mathscr{L}(x) y
$$

for all $x, y \in \mathscr{A}$.

Due to additivity of $\mathscr{L}$ and (24), we see that

$$
\begin{aligned}
3^{n} x f & (y)+3^{n} \mathscr{L}(x) y=\mathscr{L}\left(3^{n} x \cdot y\right) \\
= & \mathscr{L}\left(x \cdot 3^{n} y\right)=x f\left(3^{n} y\right)+3^{n} \mathscr{L}(x) y .
\end{aligned}
$$

So we obtain $x f(y)=x\left(f\left(3^{n} y\right) / 3^{n}\right)$; that is,

$$
\begin{aligned}
& \mu\left(x f(y)-x \frac{f\left(3^{n} y\right)}{3^{n}}, t\right)=1, \\
& \nu\left(x f(y)-x \frac{f\left(3^{n} y\right)}{3^{n}}, t\right)=0
\end{aligned}
$$

for all $x, y \in \mathscr{A}$ and $t>0$. From this, it follows that

$$
\begin{aligned}
& \mu(x f(y)-x \mathscr{L}(y), t) \\
& \geq \mu\left(x f(y)-x \frac{f\left(3^{n} y\right)}{3^{n}}, \frac{t}{2}\right) \\
& * \mu\left(x \frac{f\left(3^{n} y\right)}{3^{n}}-x \mathscr{L}(y), \frac{t}{2}\right) \\
&= \mu\left(x \frac{f\left(3^{n} y\right)}{3^{n}}-x \mathscr{L}(y), \frac{t}{2}\right), \\
& \nu(x f(y)-x \mathscr{L}(y), t) \\
& \leq\left(x f(y)-x \frac{f\left(3^{n} y\right)}{3^{n}}, \frac{t}{2}\right) \\
& \diamond \nu\left(x \frac{f\left(3^{n} y\right)}{3^{n}}-x \mathscr{L}(y), \frac{t}{2}\right) \\
&=v\left(x \frac{f\left(3^{n} y\right)}{3^{n}}-x \mathscr{L}(y), \frac{t}{2}\right)
\end{aligned}
$$

for all $x, y \in \mathscr{A}$ and $t>0$. On the other hand, we find that

$$
\begin{aligned}
\mu(x & \left.\frac{f\left(3^{n} y\right)}{3^{n}}-x \mathscr{L}(y), \frac{t}{2}\right) \\
& =\mu\left(x f\left(3^{n} y\right)-3^{n} x \mathscr{L}(y), \frac{3^{n} t}{2}\right) \\
& \geq \max \left\{\mu\left(x, \frac{3^{n} t}{4}\right), \mu\left(\frac{f\left(3^{n} y\right)}{3^{n}}-\mathscr{L}(y), \frac{t}{4}\right)\right\}, \\
\nu(x & \left.\frac{f\left(3^{n} y\right)}{3^{n}}-x \mathscr{L}(y), \frac{t}{2}\right) \\
& =v\left(x f\left(3^{n} y\right)-3^{n} x \mathscr{L}(y), \frac{3^{n} t}{2}\right) \\
& \leq \min \left\{v\left(x, \frac{3^{n} t}{4}\right), v\left(\frac{f\left(3^{n} y\right)}{3^{n}}-\mathscr{L}(y), \frac{t}{4}\right)\right\}
\end{aligned}
$$

for all $x, y \in \mathscr{A}$ and $t>0$. Taking $n \rightarrow \infty$ in (27) and (28), we obtain that

$$
\mu(x f(y)-x \mathscr{L}(y), t)=1, \quad v(x f(y)-x \mathscr{L}(y), t)=0 .
$$

Therefore,

$$
x f(y)=x \mathscr{L}(y)
$$

for all $x, y \in \mathscr{A}$, which implies that condition (19) holds.

Comparing (24) and (30), we have $\mathscr{L}(x y)=x \mathscr{L}(y)+$ $\mathscr{L}(x) y$. This completes the proof.

By considering the unit, the following result can be obtained easily from Theorem 9 . 
Corollary 10. Let $(\mathscr{A}, \mu, \nu, *, \diamond)$ be an intuitionistic fuzzy Banach algebra with unit, and let $f: \mathscr{A} \rightarrow \mathscr{A}$ be a mapping with $f(0)=0$. Assume that $\varphi$ is a function from $\mathscr{A}$ to an intuitionistic fuzzy normed space $\left(C, \mu^{\prime}, \nu^{\prime}, *, \diamond\right)$ satisfying (3), and that $\Phi$ is a function from $\mathscr{A}$ to an intuitionistic fuzzy normed space $\left(D, \mu^{\prime \prime}, \nu^{\prime \prime}, *, \diamond\right)$ satisfying (18). If $\varphi(3 x)=$ $\alpha \varphi(x)$ for some real number $\alpha$ with $0<|\alpha|<3$ and $\Phi(3 x)=$ $\beta \Phi(x)$ for some real number $\beta$ with $0<|\beta|<3$, then $f: \mathscr{A} \rightarrow$ $\mathscr{B}$ is a ring derivation.

We can also prove the preceding results for the case when $\alpha>3$ and $\beta>3$. In this case, the mapping $\mathscr{L}(x):=$ $\lim _{n \rightarrow \infty} 3^{n} f\left(3^{-n} x\right)$. In the case of intuitionistic fuzzy normed algebras with unit, we can also prove the superstability of functional equation $f(x y)=x f(y)+f(x) y$ as follows.

Theorem 11. Let $(\mathscr{A}, \mu, \nu, *, \diamond)$ be an intuitionistic fuzzy normed algebra with unit. Suppose that $f: \mathscr{A} \rightarrow \mathscr{A}$ is a mapping for which there exists a function $\Phi$ from $\mathscr{A}$ to an intuitionistic fuzzy normed space $\left(D, \mu^{\prime \prime}, \nu^{\prime \prime}, *, \diamond\right)$ satisfying (18). If $l>1$ is a fixed integer and $\Phi(l x)=\beta \Phi(x)$ for some real number $\beta$ with $0<|\beta|<l$, then $f$ satisfies the functional equation $f(x y)=x f(y)+f(x) y$ for all $x, y \in \mathscr{A}$.

Proof. Without loss of generality, we assume that $0<\beta<l$. Here, we will denote the unit by $e$. We first note that

$$
\begin{aligned}
& \mu\left(\frac{f\left(l^{n} x \cdot y\right)}{l^{n}}-x f(y)-\frac{f\left(l^{n} x\right)}{l^{n}} y, t\right) \\
& \quad=\mu\left(f\left(l^{n} x \cdot y\right)-l^{n} x \cdot f(y)-f\left(l^{n} x\right) y, l^{n} t\right) \\
& \quad \geq \max \left\{\mu^{\prime \prime}\left(\Phi(x),\left(\frac{l}{\beta}\right)^{n} \frac{t}{2}\right), \mu^{\prime \prime}\left(\Phi(y), \frac{l^{n} t}{2}\right)\right\}, \\
& \nu\left(\frac{f\left(l^{n} x \cdot y\right)}{l^{n}}-x f(y)-\frac{f\left(l^{n} x\right)}{l^{n}} y, t\right) \\
& \quad=v\left(f\left(l^{n} x \cdot y\right)-l^{n} x \cdot f(y)-f\left(l^{n} x\right) y, l^{n} t\right) \\
& \quad \leq \min \left\{v^{\prime \prime}\left(\Phi(x),\left(\frac{l}{\beta}\right)^{n} \frac{t}{2}\right), v^{\prime \prime}\left(\Phi(y), \frac{l^{n} t}{2}\right)\right\}
\end{aligned}
$$

for all $x, y \in \mathscr{A}$ and $t>0$, which mean that

$$
(\mu, v)-\lim _{n \longrightarrow \infty}\left(\frac{f\left(l^{n} x \cdot y\right)}{l^{n}}-\frac{f\left(l^{n} x\right)}{l^{n}} y\right)=x f(y)
$$

for all $x, y \in \mathscr{A}$. Secondly, we find that

$$
\begin{aligned}
& \mu\left(\frac{f\left(x \cdot l^{n} y\right)}{l^{n}}-x \frac{f\left(l^{n} y\right)}{l^{n}}-f(x) y, t\right) \\
& \quad=\mu\left(f\left(x \cdot l^{n} y\right)-x f\left(l^{n} y\right)-f(x) \cdot l^{n} y, l^{n} t\right) \\
& \quad \geq \max \left\{\mu^{\prime \prime}\left(\Phi(x), \frac{l^{n} t}{2}\right), \mu^{\prime \prime}\left(\Phi(y),\left(\frac{l}{\beta}\right)^{n} \frac{t}{2}\right)\right\},
\end{aligned}
$$

$$
\begin{aligned}
& \nu\left(\frac{f\left(x \cdot l^{n} y\right)}{l^{n}}-x \frac{f\left(l^{n} y\right)}{l^{n}}-f(x) y, t\right) \\
& \quad=v\left(f\left(x \cdot l^{n} y\right)-x f\left(l^{n} y\right)-f(x) \cdot l^{n} y, l^{n} t\right) \\
& \quad \leq \min \left\{v^{\prime \prime}\left(\Phi(x), \frac{l^{n} t}{2}\right), \nu^{\prime \prime}\left(\Phi(y),\left(\frac{l}{\beta}\right)^{n} \frac{t}{2}\right)\right\}
\end{aligned}
$$

for all $x, y \in \mathscr{A}$ and $t>0$. This implies that

$$
(\mu, v)-\lim _{n \longrightarrow \infty}\left(\frac{f\left(x \cdot l^{n} y\right)}{l^{n}}-x \frac{f\left(l^{n} y\right)}{l^{n}}\right)=f(x) y
$$

for all $x, y \in \mathscr{A}$.

We also know that

$$
\begin{aligned}
\mu(f(x y)- & x f(y)-f(x) y, t) \\
\geq \mu( & f(x y)-\frac{f\left(l^{n} e \cdot x \cdot y\right)}{l^{n}}+\frac{f\left(l^{n} e\right)}{l^{n}} x y \\
& -x f(y)+\frac{f\left(l^{n} x \cdot y\right)}{l^{n}}-\frac{f\left(l^{n} x\right)}{l^{n}} y-f(x) y \\
& \left.+\frac{f\left(l^{n} x \cdot y\right)}{l^{n}}-x \frac{f\left(l^{n} y\right)}{l^{n}}, \frac{t}{2}\right) \\
* \mu & \left(\frac{f\left(l^{n} e \cdot x \cdot y\right)}{l^{n}}-\frac{f\left(l^{n} e\right)}{l^{n}} x y-\frac{f\left(l^{n} x \cdot y\right)}{l^{n}}\right. \\
& \left.+\frac{f\left(l^{n} x\right)}{l^{n}} y-\frac{f\left(l^{n} x \cdot y\right)}{l^{n}}+x \frac{f\left(l^{n} y\right)}{l^{n}}, \frac{t}{2}\right), \\
v(f(x y)- & x f(y)-f(x) y, t) \\
\geq v( & f(x y)-\frac{f\left(l^{n} e \cdot x \cdot y\right)}{l^{n}}+\frac{f\left(l^{n} e\right)}{l^{n}} x y \\
& +x f(y)+\frac{f\left(l^{n} x \cdot y\right)}{l^{n}}-\frac{f\left(l^{n} x\right)}{l^{n}} y-f(x) y \\
& \left.+\frac{f\left(l^{n} x \cdot y\right)}{l^{n}}-x \frac{f\left(l^{n} y\right)}{l^{n}}, \frac{t}{2}\right) \\
\left.l^{n} y-\frac{f\left(l^{n} e \cdot x \cdot y\right)}{l^{n}}-\frac{f\left(l^{n} e\right)}{l^{n}} x y-\frac{f\left(l^{n} x \cdot y\right)}{l^{n}}+x \frac{f\left(l^{n} y\right)}{l^{n}}, \frac{t}{2}\right), &
\end{aligned}
$$


for all $x, y \in \mathscr{A}$ and $t>0$. On the other hand, we see that

$$
\begin{aligned}
& v\left(\frac{f\left(l^{n} e \cdot x \cdot y\right)}{l^{n}}-\frac{f\left(l^{n} e\right)}{l^{n}} x y-\frac{f\left(l^{n} x \cdot y\right)}{l^{n}}\right. \\
& \left.+\frac{f\left(l^{n} x\right)}{l^{n}} y-\frac{f\left(l^{n} x \cdot y\right)}{l^{n}}+x \frac{f\left(l^{n} y\right)}{l^{n}}, \frac{t}{2}\right) \\
& =v\left(\left(f\left(l^{n} e \cdot x\right)-l^{n} e \cdot f(x)-f\left(l^{n} e\right) x\right) y\right. \\
& \left.+l^{n} f(x) y+x f\left(l^{n} y\right)-f\left(l^{n} x \cdot y\right), \frac{l^{n} t}{2}\right) \\
& \leq \min \left\{v \left(\left(f\left(l^{n} e \cdot x\right)-l^{n} e \cdot f(x)\right.\right.\right. \\
& \left.\left.\quad-f\left(l^{n} e\right) x\right) y, \frac{l^{n} t}{4}\right) \\
& \left.\diamond v\left(l^{n} f(x) y+x f\left(l^{n} y\right)-f\left(l^{n} x \cdot y\right), \frac{l^{n} t}{4}\right)\right\}
\end{aligned}
$$$$
\mu\left(f(x y)-\frac{f\left(l^{n} e \cdot x \cdot y\right)}{l^{n}}+\frac{f\left(l^{n} e\right)}{l^{n}} x y-x f(y)\right.
$$$$
+\frac{f\left(l^{n} x \cdot y\right)}{l^{n}}-\frac{f\left(l^{n} x\right)}{l^{n}} y-f(x) y
$$$$
\left.+\frac{f\left(l^{n} x \cdot y\right)}{l^{n}}-x \frac{f\left(l^{n} y\right)}{l^{n}}, \frac{t}{2}\right)
$$$$
\geq \mu\left(f(x y)-\left(\frac{f\left(l^{n} e \cdot x \cdot y\right)}{l^{n}}-\frac{f\left(l^{n} e\right)}{l^{n}} x y\right), \frac{t}{6}\right)
$$$$
\text { * } \mu\left(\left(\frac{f\left(l^{n} x \cdot y\right)}{l^{n}}-\frac{f\left(l^{n} x\right)}{l^{n}} y\right)-x f(y), \frac{t}{6}\right)
$$$$
\text { * } \mu\left(\left(\frac{f\left(l^{n} x \cdot y\right)}{l^{n}}-x \frac{f\left(l^{n} y\right)}{l^{n}}\right)-f(x) y, \frac{t}{6}\right),
$$$$
v\left(f(x y)-\frac{f\left(l^{n} e \cdot x \cdot y\right)}{l^{n}}+\frac{f\left(l^{n} e\right)}{l^{n}} x y\right.
$$$$
-x f(y)+\frac{f\left(l^{n} x \cdot y\right)}{l^{n}}-\frac{f\left(l^{n} x\right)}{l^{n}} y
$$$$
\left.-f(x) y+\frac{f\left(l^{n} x \cdot y\right)}{l^{n}}-x \frac{f\left(l^{n} y\right)}{l^{n}}, \frac{t}{2}\right)
$$$$
\leq v\left(f(x y)-\left(\frac{f\left(l^{n} e \cdot x \cdot y\right)}{l^{n}}-\frac{f\left(l^{n} e\right)}{l^{n}} x y\right), \frac{t}{6}\right)
$$$$
\diamond v\left(\left(\frac{f\left(l^{n} x \cdot y\right)}{l^{n}}-\frac{f\left(l^{n} x\right)}{l^{n}} y\right)-x f(y), \frac{t}{6}\right)
$$$$
\diamond v\left(\left(\frac{f\left(l^{n} x \cdot y\right)}{l^{n}}-x \frac{f\left(l^{n} y\right)}{l^{n}}\right)-f(x) y, \frac{t}{6}\right)
$$

for all $x, y \in \mathscr{A}$ and $t>0$. In particular, we obtain the following:

$$
\begin{gathered}
\mu\left(\left(f\left(l^{n} e \cdot x\right)-l^{n} e \cdot f(x)-f\left(l^{n} e\right) x\right) y, \frac{l^{n} t}{4}\right) \\
\geq \max \left\{\mu \left(f\left(l^{n} e \cdot x\right)-l^{n} e \cdot f(x)\right.\right. \\
\left.\left.-f\left(l^{n} e\right) x, \frac{l^{n} t}{8}\right), \mu\left(y, \frac{l^{n} t}{8}\right)\right\}, \\
\nu\left(\left(f\left(l^{n} e \cdot x\right)-l^{n} e \cdot f(x)-f\left(l^{n} e\right) x\right) y, \frac{l^{n} t}{4}\right) \\
\leq \min \left\{v \left(f\left(l^{n} e \cdot x\right)-l^{n} e \cdot f(x)\right.\right. \\
\left.\left.\quad-f\left(l^{n} e\right) x, \frac{l^{n} t}{8}\right), v\left(y, \frac{l^{n} t}{8}\right)\right\}, \\
\mu\left(f\left(l^{n} e \cdot x\right)-l^{n} e \cdot f(x)-f\left(l^{n} e\right) x, \frac{l^{n} t}{8}\right) \\
\quad \geq \max \left\{\mu^{\prime \prime}\left(\Phi(e),\left(\frac{l}{\beta}\right)^{n} \frac{t}{16}\right), \mu^{\prime \prime}\left(\Phi(x), \frac{l^{n} t}{16}\right)\right\}, \\
\nu\left(f\left(l^{n} e \cdot x\right)-l^{n} e \cdot f(x)-f\left(l^{n} e\right) x, \frac{l^{n} t}{8}\right) \\
\quad \leq \min \left\{v^{\prime \prime}\left(\Phi(e),\left(\frac{l}{\beta}\right)^{n} \frac{t}{16}\right), v^{\prime \prime}\left(\Phi(x), \frac{l^{n} t}{16}\right)\right\}
\end{gathered}
$$

for all $x, y \in \mathscr{A}$ and $t>0$. Also, we arrive at

$$
\begin{aligned}
& \mu\left(l^{n} f(x) y+x f\left(l^{n} y\right)-f\left(l^{n} x \cdot y\right), \frac{l^{n} t}{4}\right) \\
& \quad=\mu\left(f\left(x \cdot l^{n} y\right)-x f\left(l^{n} y\right)-l^{n} f(x) y, \frac{l^{n} t}{4}\right) \\
& \quad \geq \max \left\{\mu^{\prime \prime}\left(\Phi(x), \frac{l^{n} t}{8}\right), \mu^{\prime \prime}\left(\Phi(y),\left(\frac{l}{\beta}\right)^{n} \frac{t}{8}\right)\right\},
\end{aligned}
$$




$$
\begin{aligned}
& v\left(l^{n} f(x) y+x f\left(l^{n} y\right)-f\left(l^{n} x \cdot y\right), \frac{l^{n} t}{4}\right) \\
& \quad=v\left(f\left(x \cdot l^{n} y\right)-x f\left(l^{n} y\right)-l^{n} f(x) y, \frac{l^{n} t}{4}\right) \\
& \quad \leq \min \left\{v^{\prime \prime}\left(\Phi(x), \frac{l^{n} t}{8}\right), v^{\prime \prime}\left(\Phi(y),\left(\frac{l}{\beta}\right)^{n} \frac{t}{8}\right)\right\} .
\end{aligned}
$$

Letting $n \rightarrow \infty$ in (35)-(39) with (32) and (34), we get the desired result. This completes the proof.

We remark that we can also verify Theorem 11 for the case when $\beta>l$.

\section{Acknowledgments}

The authors would like to thank the referees for giving useful suggestions and for the improvement of this paper. This research was supported by Basic Science Research Program through the National Research Foundation of Korea (NRF) funded by the Ministry of Education (no. 2010-002338 and no. 2013R1A1A2A10004419). This research was also supported by the National Research Foundation of Korea (NRF) funded by the Ministry of Science, ICT and Future Planning (no. 2012R1A1A2021721).

\section{References}

[1] S. M. Ulam, A Collection of Mathematical Problems, Interscience, New York, NY, USA, 1960.

[2] D. H. Hyers, "On the stability of the linear functional equation," Proceedings of the National Academy of Sciences of the United States of America, vol. 27, pp. 222-224, 1941.

[3] T. Aoki, "On the stability of the linear transformation in Banach spaces," Journal of the Mathematical Society of Japan, vol. 2, pp. 64-66, 1950.

[4] T. M. Rassias, "On the stability of the linear mapping in Banach spaces," Proceedings of the American Mathematical Society, vol. 72, no. 2, pp. 297-300, 1978.

[5] R. Badora, "On approximate ring homomorphisms," Journal of Mathematical Analysis and Applications, vol. 276, no. 2, pp. 589597, 2002.

[6] D. G. Bourgin, "Approximately isometric and multiplicative transformations on continuous function rings," Duke Mathematical Journal, vol. 16, pp. 385-397, 1949.

[7] R. Badora, "On approximate derivations," Mathematical Inequalities \& Applications, vol. 9, no. 1, pp. 167-173, 2006.

[8] A. K. Mirmostafaee and M. S. Moslehian, "Fuzzy almost quadratic functions," Results in Mathematics, vol. 52, no. 1-2, pp. 161177, 2008.

[9] A. K. Mirmostafaee and M. S. Moslehian, "Fuzzy versions of Hyers-Ulam-Rassias theorem," Fuzzy Sets and Systems, vol. 159, no. 6, pp. 720-729, 2008.

[10] S. A. Mohiuddine, "Stability of Jensen functional equation in intuitionistic fuzzy normed space," Chaos, Solitons \& Fractals, vol. 42, no. 5, pp. 2989-2996, 2009.

[11] S. A. Mohiuddine, M. Cancan, and H. Şevli, "Intuitionistic fuzzy stability of a Jensen functional equation via fixed point technique," Mathematical and Computer Modelling, vol. 54, no. 9-10, pp. 2403-2409, 2011.
[12] S. A. Mohiuddine and H. Ševli, "Stability of Pexiderized quadratic functional equation in intuitionistic fuzzy normed space," Journal of Computational and Applied Mathematics, vol. 235, no. 8, pp. 2137-2146, 2011.

[13] M. Mursaleen and S. A. Mohiuddine, "On stability of a cubic functional equation in intuitionistic fuzzy normed spaces," Chaos, Solitons \& Fractals, vol. 42, no. 5, pp. 2997-3005, 2009.

[14] R. Saadati and J. H. Park, "On the intuitionistic fuzzy topological spaces," Chaos, Solitons \& Fractals, vol. 27, no. 2, pp. 331-344, 2006.

[15] B. Dinda, T. K. Samanta, and U. K. Bera, "Intuitionistic fuzzy Banach algebra," Bulletin of Mathematical Analysis and Applications, vol. 3, no. 3, pp. 273-281, 2011. 


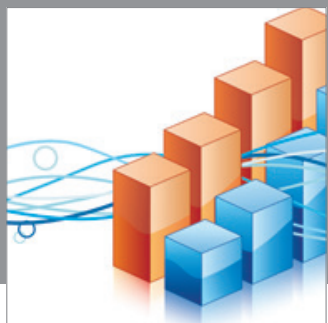

Advances in

Operations Research

mansans

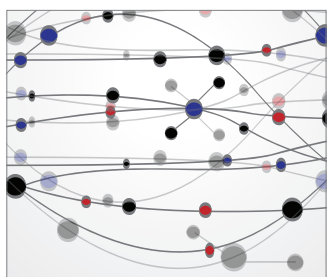

The Scientific World Journal
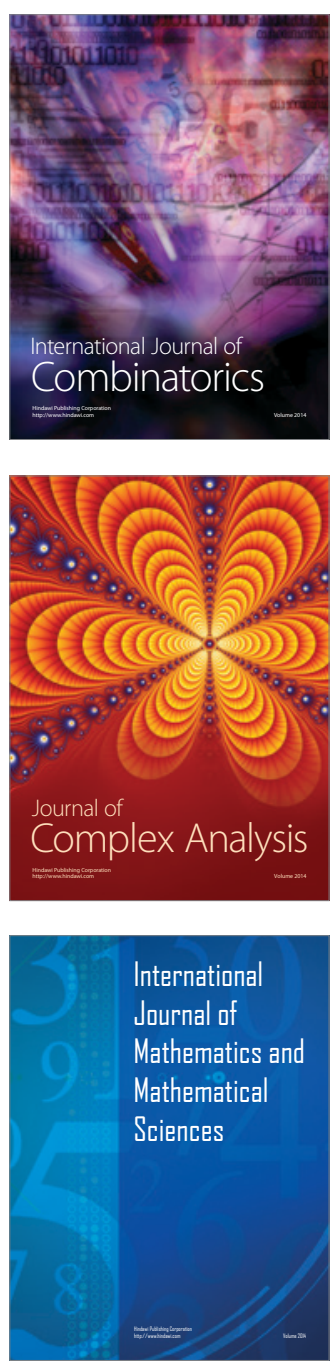
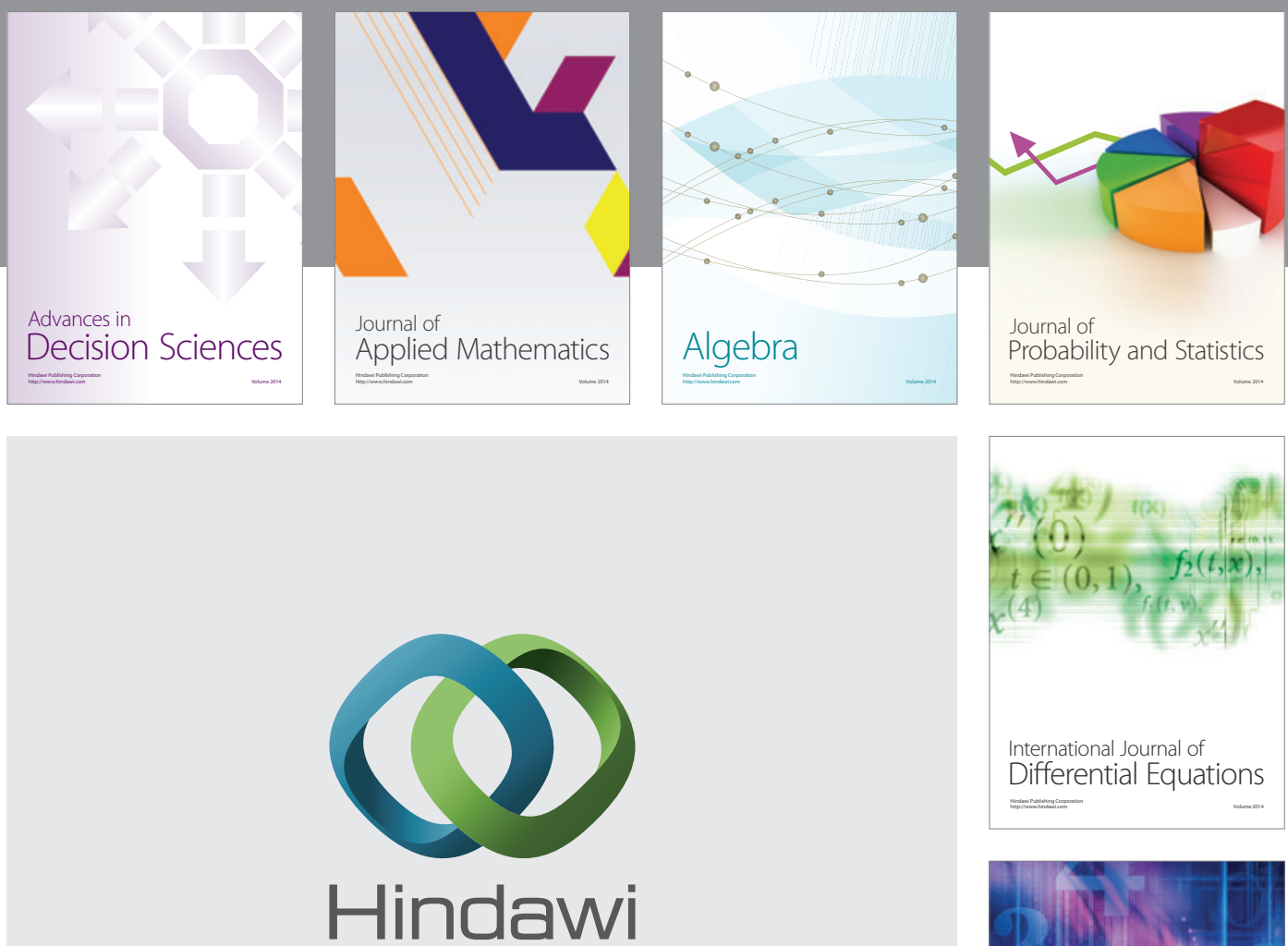

Submit your manuscripts at http://www.hindawi.com
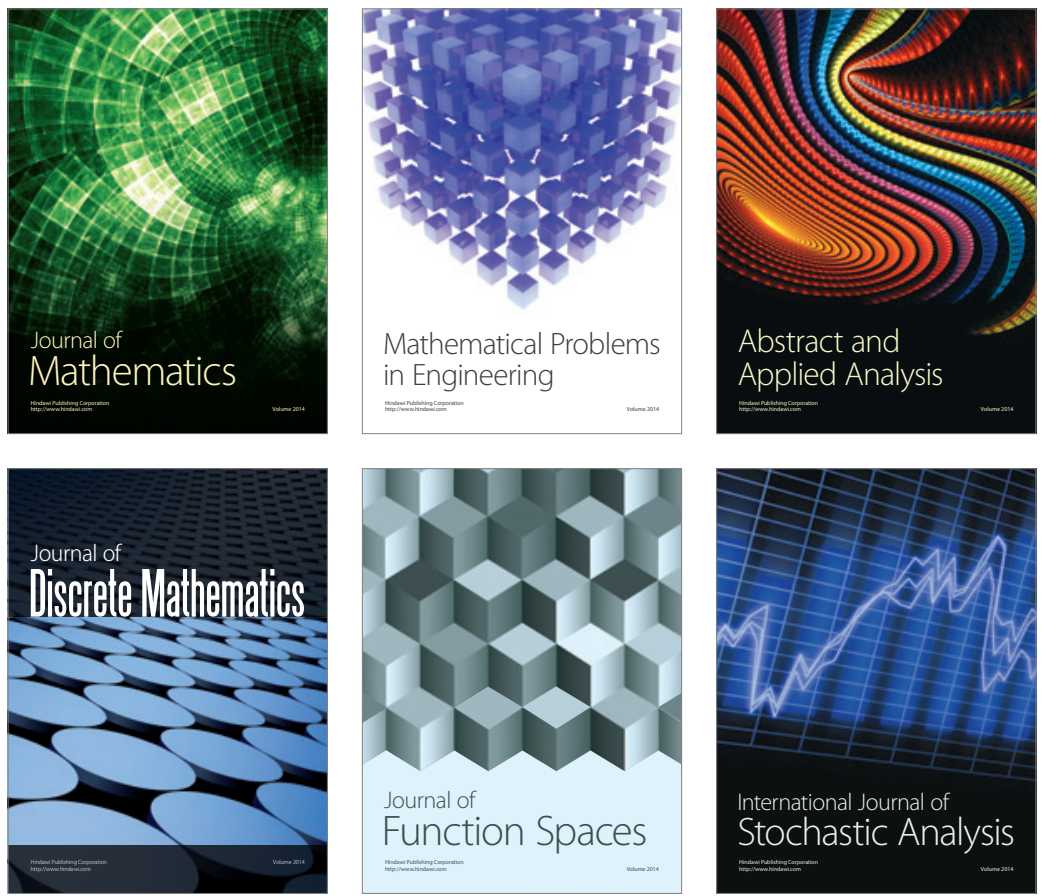

Journal of

Function Spaces

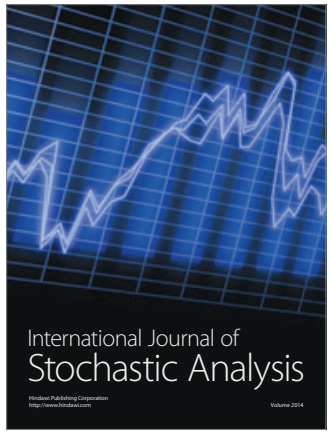

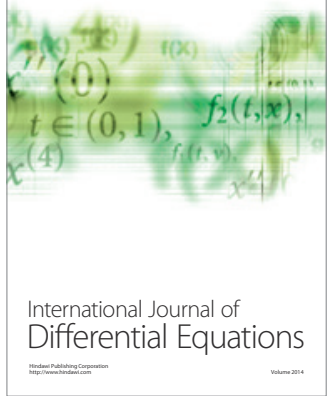
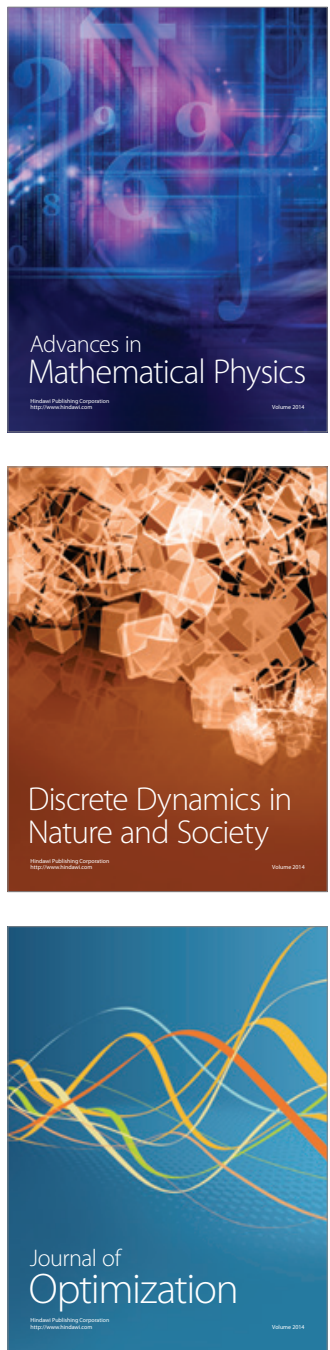